\title{
The source and role of RANTES in interstitial lung disease
}

\author{
M. Petrek+, P. Pantelidis*, A.M. Southcott*, P. Lympany*, P. Safranek+, C.M. Black**, \\ V. Kolek ${ }^{++}$, E. Weigl+, R.M. du Bois*
}

The source and role of RANTES in interstitial lung disease. M. Petrek, P. Pantelidis, A.M. Southcott, P. Lympany, P. Safranek, C.M. Black, V. Kolek, E. Weigl, R.M. du Bois. (CERS Journals Ltd 1997.

ABSTRACT: The chemokine "regulated on activation, normal T-cell expressed and secreted" (RANTES) is a potent eosinophil and lymphocyte attractant with particular preference for CD45RO+ T-cells and eosinophils. These cells accumulate in the lungs of patients with sarcoidosis and fibrosing alveolitis. The purpose of this study was to determine whether RANTES mediates the inflammatory cell influx in these diffuse lung diseases.

Cell types and number of bronchoalveolar cells expressing RANTES protein were investigated by immunocytochemistry using lavage cells obtained from 22 patients and 11 control subjects. Subsequently, RANTES messenger ribonucleic acid (mRNA) was semiquantitated using reverse transcription polymerase chain reaction (RT-PCR) methodology in unseparated lavage cell pellets in 26 patients and 13 control subjects. Cells expressing RANTES mRNA were identified by in situ hybridization.

RANTES protein expression in lower respiratory tract (LRT) cells was identified in all study groups. The percentage of RANTES+ lavage cells in sarcoidosis was higher than in controls. RANTES was localized in the cytoplasm, mainly in alveolar macrophages (CD68+ cells) in sarcoidosis, and both in alveolar macrophages and eosinophils in fibrosing alveolitis. The same cell types which expressed RANTES protein expressed RANTES mRNA, as assessed by in situ hybridization. Sarcoidosis patients had higher levels of RANTES mRNA than the other groups. RANTES protein was higher in individuals with abnormal lymphocyte numbers: RANTES protein and mRNA expression was significantly correlated with lavage CD45RO+ lymphocyte numbers.

These results indicate that RANTES may mediate T-lymphocyte influx in diffuse lung disease, particularly sarcoidosis. Moreover, they suggest that the cellular source of RANTES is the alveolar macrophage in sarcoidosis, and both macrophages and eosinophils in fibrosing alveolitis.

Eur Respir J 1997; 10: 1207-1216.
*Interstitial Lung Disease Unit, Royal Brompton Hospital and National Heart and Lung Institute, London, UK. +Dept of Immunology, Medical Faculty of Palacky University, Olomouc, Czech Republic. **Dept of Rheumatology, Royal Free Hospital, London, UK. ++Dept of Respiratory Medicine, University Hospital Olomouc, Czech Republic.

Correspondence: R.M. du Bois

Royal Brompton Hospital

Emmanuel Kaye Building

Manresa Road

London SW3 6LR

UK

Keywords: Chemokine RANTES

eosinophils

fibrosing alveolitis

sarcoidosis

T-lymphocytes

Received: July 311996

Accepted after revision January 181997

MP is the recipient of a European Community research fellowship. This work was supported, in part, by funds from the Czech grant agencies IGA MZ CR and FRVS.
Interstitial lung disease (ILD) encompasses a large number of disorders characterized by distinct cellular infiltrates in the acinar regions of the lung. The most frequently diagnosed ILDs, sarcoidosis and fibrosing alveolitis, are chronic conditions with distinct pathogenesis, prognosis and composition of inflammatory infiltrates. In sarcoidosis, increased numbers of CD4+ CD45RO+ "memory" T-lymphocytes accumulate within alveolar spaces as a consequence of triggering of the immune response by an, as yet unknown, antigen $[1,2]$. The infiltrate in fibrosing alveolitis (FA) is primarily interstitial, but increased numbers of inflammatory cells can also be found in bronchoalveolar lavage (BAL) fluid $[3,4]$ : eosinophils and lymphocytes are present in the earlier phases of FA, neutrophil infiltration becomes prominent in the later stages of the disease. The majority of the lymphocytes infiltrating the interstitium also express the CD45RO surface marker [5].

The mechanisms responsible for the migration of inflammatory cells from the vasculature to the lung in
ILD are not known, but members of the growing family of chemotactic cytokines (chemokines) [6] are prime candidates. In a previous study from our group, we identified interleukin-8 (IL-8) as a possible mediator of neutrophil alveolitis in fibrosing alveolitis associated with systemic sclerosis [7]. Others have suggested that the chemokines, macrophage inflammatory protein-1a (MIP1a), monocyte chemoattractant protein (MCP-1) and IL8 , contribute to the influx of monocytes and neutrophils to the lung in sarcoidosis and fibrosing alveolitis [8,9]. However, the role of lymphocyte or eosinophil attractant chemokines in these two interstitial lung diseases has not been investigated.

The chemokine "regulated on activation, normal Tcell expressed and secreted" (RANTES) [10] has been shown in vitro to attract T-lymphocytes, particularly CD45RO+ T-cells [11, 12], and is also an eosinophil attractant and activator [13]. We have, therefore, hypothesized that RANTES may also mediate recruitment of T-lymphocytes and eosinophils to the lower respiratory 
tract in vivo in ILD, and that RANTES expression may be upregulated in patients with lymphocytic and/or eosinophilic alveolitis.

To address this hypothesis, we have investigated RANTES messenger ribonucleic acid (mRNA) and protein expression in lower respiratory tract (LRT) cells of patients with sarcoidosis and patients with FA, and compared it to the expression in control subjects. We have been particularly interested in evaluating which cell types in the LRT express RANTES, and whether there is any relationship between RANTES expression and the number of its target cells (lymphocytes and eosinophils) present on the epithelial lining surface.

\section{Methods}

\section{Study population}

Subjects for the analysis of cell-associated RANTES protein. In the first stage of the study, expression of RANTES protein was investigated by immunocytochemistry in BAL cells from 33 subjects: patients with fibrosing alveolitis (FA), patients with pulmonary sarcoidosis (S) and control subjects $(C)$. The FA group $(n=12$; aged $45 \pm 3$ (SEM) yrs; 3 smokers; 7 receiving treatment) included seven patients with cryptogenic fibrosing alveolitis (CFA) and five patients in whom fibrosing alveolitis was associated with systemic sclerosis (FASSc). The diagnosis of fibrosing alveolitis was based on clinical, radiographic (chest radiography and high resolution computed tomography (HRCT)) and physiological data. FA was confirmed by biopsy in 6 of the 12 individuals. The sarcoidosis group comprised 10 patients (aged $45 \pm 3$ yrs; 2 smokers; 3 receiving treatment). The diagnosis of sarcoidosis was based on characteristic histological appearances of biopsy samples and/or on a positive Kveim test. The control group $(n=11$; aged $40 \pm 4$ yrs; 3 smokers; none receiving treatment) included nine healthy volunteers with no evidence of respiratory disease $(\mathrm{N})$; and two patients with systemic sclerosis without any evidence of FA (SScnoFA), based on clinical, physiological, and radiographic (including HRCT) evidence.

Subjects for mRNA analysis. In the second stage of the study, reverse transcription-polymerase chain reaction (RTPCR) methodology was utilized to determine RANTES mRNA expression in unseparated BAL cells from 39 subjects: 20 patients with fibrosing alveolitis (7 CFA and 13 FASSc; aged $46 \pm 2$ yrs; no smokers; 13 receiving treatment); six patients with pulmonary sarcoidosis (aged 47 2 yrs; no smokers; 2 receiving treatment); and 13 control subjects (9 $\mathrm{N}$ and 4 SScnoFA; aged $47 \pm 3$ yrs; 1 smoker; none receiving treatment).

In situ hybridization (ISH) experiments for detection of RANTES mRNA in individual BAL cells were performed in three randomly selected patients with CFA and four patients with sarcoidosis.

Approval for studies of human subjects was obtained from the local Ethics Committee.

\section{Bronchoalveolar lavage (BAL)}

LRT cells from normal individuals and patients were recovered by BAL, which was performed according to our standard protocol. In brief, during flexible fibreoptic bronchoscopy, $100 \mathrm{~mL}$ prewarmed saline was instilled into subsegmental bronchi in three sites: right middle lobe; right lower lobe; and lingula. The cells were separated from the recovered BAL fluid by centrifugation at $200 \times \mathrm{g}, 4^{\circ} \mathrm{C}$ for $7 \mathrm{~min}$. The supernatant obtained after the centrifugation was collected and stored at $-20^{\circ} \mathrm{C}$ until further analysis. The cell pellet was washed twice and resuspended in RPMI-1640 medium, and total cell recovery estimated. Following preparation of cytospin slides, the remaining cells were pelleted, lysed in guanidine isothiocyanate (GITC) lysis buffer with 1\% 2-mercaptoethanol ( $1 \mathrm{~mL}$ of buffer per $10^{7}$ cells) and stored at $-80^{\circ} \mathrm{C}$ until ribonucleic acid (RNA) extraction.

\section{$B A L$ differential count and determination of lymphocyte subsets}

Cytocentrifuge preparations were made using Cytospin2 (Shandon Instr., Sewickly, USA) and utilized for determination of BAL differential counts and, after fixation with acetone-chloroform, for immunocytochemical analysis of RANTES protein expression and determination of CD45RO+ or CD45RA+ lymphocytes.

Differential cell counts were determined in duplicate from cytospin preparations: 200 May-Grünwald Giemsa stained cells were counted and the percentages and total numbers of each cell type were calculated.

CD45RO+ lymphocytes were identified using an anti-CD45RO monoclonal antibody (Leu45RO; BectonDickinson, Heidelberg, Germany). Visualization of positively stained cells was achieved using the alkaline phosphatase/anti-alkaline phosphatase (APAAP) immunocytochemical method [14], employing a rabbit antimouse immunoglobulin $\mathrm{G}$ (IgG) as a bridging antibody and fast red as a chromogen; the slides were counterstained with haematoxylin. Before immunostaining, nonspecific binding sites were blocked using $20 \%$ normal rabbit serum (all reagents for APAAP: Dako, Glostrup, Denmark; haematoxylin: BDH, Poole, UK). For all subjects, duplicate slides were processed and 100 lymphocytes were scored on each slide. BAL (total and differential) CD45RO+ lymphocyte counts were derived from the percentage of lymphocytes which were CD45RO+ and BAL lymphocyte total numbers and percentages; (total CD45RO+ lymphocyte count $=\mathrm{BAL}$ total lymphocyte count $\times$ CD45RO+ percentage/100; differential $\mathrm{CD} 45 \mathrm{RO}+$ count $=$ percentage of lavage cells which were lymphocytes $\times$ CD45RO+ percentage/100).

To ensure the reciprocity of CD45RO and CD45RA staining, cytospin preparations from nine randomly selected subjects (five patients and four normals) were used to identify CD45RA+ cells using an anti-CD45RA monoclonal antibody (clone MEM-93; Czech Academy of Science, Prague) followed by APAAP immunostaining.

\section{Determination of cell-associated RANTES protein by immunocytochemistry}

To identify cell-associated RANTES protein, cytospins of bronchoalveolar lavage fluid (BALF) cells were incubated with anti-RANTES monoclonal antibody (R\&D 
Systems, Abingdon, UK); in parallel slides, the antibody was substituted by an irrelevant negative control monoclonal antibody of the same isotype (DAKO, Glostrup, Denmark). As an additional control for the specificity of the staining, the anti-RANTES antibody was preincubated with $1 \mathrm{ng} \cdot \mathrm{mL}^{-1}$ of recombinant human RANTES (R\&D Systems). To visualize the specific binding, biotinylated rabbit anti-mouse $\mathrm{F}\left(\mathrm{ab}^{\prime}\right)_{2}$ antibody was applied to the slides, followed by streptavidin-biotin-complexes conjugated to horseradish peroxidase (DAKO); 3,9 amino-ethyl-carbazole (Sigma, Minneapolis, USA) was used as a chromogen. Endogeneous peroxidase activity was blocked using peroxidase blocking agent (DAKO). At least 200 cells were counted by two independent observers. The results were expressed: 1) as a percentage of positively stained cells of all BAL leucocytes; and 2) as a percentage of positive macrophages, lymphocytes and eosinophils of the total number of the respective cell type. The identity of the RANTES+ cells was confirmed by parallel staining of cytospins with antiCD68 (DAKO; macrophage marker), anti-CD2 (lymphocyte marker), and visualized as for RANTES. Quantitative morphometry of RANTES+ cells was compared with that of CD68+ and CD2+ cells.

\section{RNA extraction and reverse transcription}

Total cellular RNA was isolated using the single step acid-GITC method [15]. The RNA pellet was dissolved in $50 \mu \mathrm{L}$ of sterile deionized water, $40 \mathrm{U}$ of human placental ribonuclease (RNAse) inhibitor (Promega, Southampton, UK) was added, and the samples were stored at $-80^{\circ} \mathrm{C}$ until further use.

The complementary deoxyribonucleic acid (cDNA) for PCR amplification was obtained by reverse transcription of mRNA using oligo(dT) primer [16]. Each reaction contained $1 \mu \mathrm{g}$ RNA, $0.4 \mu \mathrm{g}$ oligo $(\mathrm{dT})_{15}$ primer, $50 \mathrm{mM} \mathrm{KCl}, 10 \mathrm{mM}$ Tris- $\mathrm{HCl}$ (pH 9.0), 0.1\% Triton$\mathrm{X} 100,5 \mathrm{mM} \mathrm{MgCl}, 1 \mathrm{mM}$ each deoxynucleotide triphosphate (dNTP), $20 \mathrm{U}$ Avian myeloblastasis virus (AMV) reverse transcriptase HC (Promega) and 20-40 $\mathrm{U}$ placental RNAse inhibitor, in a total reaction volume of $40 \mu \mathrm{L}$. After incubation at $42^{\circ} \mathrm{C}$ for $60 \mathrm{~min}$, the samples were heated to $80^{\circ} \mathrm{C}$ for $10 \mathrm{~min}$ to terminate the reaction. The resulting cDNA was diluted 10 fold in sterile water, stored at $4^{\circ} \mathrm{C}$ and used within 4 weeks. To detect the presence of contaminating genomic DNA, PCR was performed with histidyl transfer ribonucleic acid (tRNA) synthetase specific primers [17].

\section{Polymerase chain reaction}

PCR reactions were performed with specific oligonucleotide primers designed from the published cDNA sequences of RANTES [10] and $\beta$-actin [18]. RANTES: 5'TCATTGCTACTGCCCTCTGC3' (sense, bases 61-80); 5'CCTAGCTCATCTCCAAAGAG3' (antisense, bases 284-303). $\beta$-actin: 5'TCCTGTGGCATCCACGAAACT3' (sense, bases 852-872); 5'GAAGCATTTGCGGTGGACGAT3' (antisense, bases 1146-1166). The predicted sizes of the amplified RANTES and $\beta$-actin cDNA products were 243 and 315 base pairs (bp), respectively.
The PCR reaction mix contained the forward and reverse primers $(0.25 \mathrm{mM}$ each $)$, dNTPs $(0.2 \mathrm{mM}$ each $)$, $10 \mathrm{mM}$ Tris- $\mathrm{HCl}$ (pH 8.3), $50 \mathrm{mM} \mathrm{KCl}, 1.5 \mathrm{mM} \mathrm{MgCl}$, $0.1 \mathrm{mg} \cdot \mathrm{mL}$ gelatine, $1.5 \mathrm{U}$ Taq polymerase (Boehringer Mannheim, Lewes, UK) and $10 \mu \mathrm{L}$ cDNA, in a total reaction volume of $30 \mu \mathrm{L}$. After initial denaturation (5 min at $95^{\circ} \mathrm{C}$ ), a range (20-36) of amplification cycles was performed using a PHC3 thermocycler (Techne, Cambridge, UK). One cycle consisted of denaturation at $95^{\circ} \mathrm{C}$ for $45 \mathrm{~s}$, annealing at $55^{\circ} \mathrm{C}$ for $45 \mathrm{~s}$ and extension at $72^{\circ} \mathrm{C}$ for $90 \mathrm{~s}$. After the last cycle, final extension $\left(10 \mathrm{~min}\right.$ at $\left.72^{\circ} \mathrm{C}\right)$ was carried out and the reaction was terminated by chilling to $4^{\circ} \mathrm{C}$. A negative control reaction containing all components except template cDNA was set up with every PCR experiment.

To define the optimum PCR conditions, a number of preliminary studies were performed: firstly, kinetic studies of RANTES and $\beta$-actin cDNA amplification determined the optimal number of amplification cycles for each gene, so that the product was within the exponential phase of the amplification process [19]; secondly, two randomly selected cDNA samples (one control and one patient sample) were amplified on four different days and showed no significant difference in variance between assays of the two samples; and thirdly, intra-assay variability was assessed in triplicate amplification of two cDNA samples (one control and one patient) on the same day, and no significant difference in variance was observed (data not shown).

Identification of PCR products, measurements and quantification of RANTES mRNA expression

The amplification products were visualized by ethidium bromide fluorescence after $3 \%$ agarose gel electrophoresis.

To verify that the bands obtained represented the specific RANTES and $\beta$-actin amplification products, the size of the bands was determined using a low molecular weight marker (pBR 322 DNA/Hae III; Boehringer Mannheim). The sizes were then compared with those predicted from the locations of the primers within RANTES or $\beta$-actin cDNA sequences.

For measurements of PCR products, the negative images of the gels on Polaroid type 665 film were scanned on a laser densitometer, and the optical density (OD) values for the individual products were obtained. RANTES mRNA expression was quantified by normalizing RANTES expression to $\beta$-actin, a gene expressed constitutively with little variability; similar quantitative PCR has previously been applied to control for variability in the amount of input RNA in studies of chemokine mRNA expression $[7,20]$. The RANTES/ $\beta$-actin optical density ratios (ODRs) were derived for each subject by comparison of optical densities of RANTES with the optical density of $\beta$-actin amplification products $(\mathrm{ODR}=\mathrm{OD}$ RANTES/OD $\beta$-actin).

\section{In situ hybridization for RANTES mRNA (ISH)}

ISH was performed on cytospins of BALF cells fixed with $4 \%$ paraformaldehyde, using an oligonucleotide 
probe cocktail to human RANTES (R\&D Systems, Abingdon, UK); the cocktail consisted of three digoxigenin-labelled probes specific to different regions of the RANTES gene. Prior to application of the probe, negative control slides were pretreated with RNAse A, to confirm the specificity of the probe for RNA and to exclude any artefacts which might arise from nonspecific binding during the detection procedure. The hybridization of the probe to RNA was visualized using anti-digoxigenin $\mathrm{F}\left(\mathrm{ab} \mathrm{b}^{\prime}\right)_{2}$ polyclonal antibody conjugated to alkaline phosphatase (Boehringer Mannheim, Lewes, UK); the binding was visualized by a nitroblue tetrazolium (NBT)/5-bromo-4-chloro-3-indolyl phosphate (BCIP) substrate system and the slides were counterstained with nuclear red. The slides were evaluated for characterization of the cellular types expressing RANTES mRNA by two independent observers.

\section{Statistical analysis}

Mann-Whitney U-test was employed to test for differences in RANTES mRNA or protein expression between the control and the disease groups. Spearman's rank correlation was used to assess the association between RANTES mRNA or protein data, and BAL cell counts and lymphocyte subsets. A p-value less than 0.05 was considered statistically significant.

\section{Results}

\section{RANTES protein expression in bronchoalveolar lavage cells}

RANTES protein was found to be expressed in BAL cells from all but one of the 33 subjects. The percentage of RANTES+ BAL cells in sarcoidosis (median 24\%, range $1-41 \%$ ) was higher than that in controls (median $7 \%$, range $0-28 \%)(\mathrm{p}<0.05)$ (fig. 1). The percentage of RANTES+ BAL cells in FA (median 12\%, range 1-28\%) did not differ from controls.

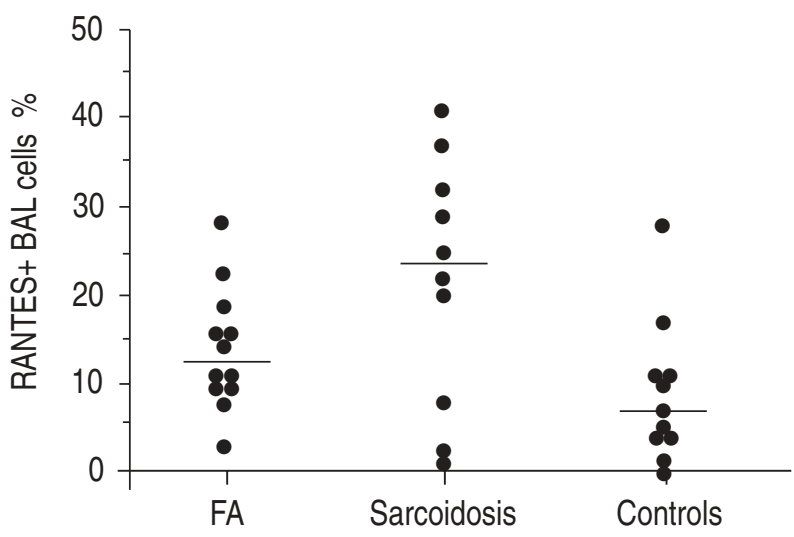

Fig. 1. - RANTES protein expression in BAL cells of patients with fibrosing alveolitis $(n=12)$, sarcoidosis $(n=10)$, and controls $(n=11)$. Cell-associated RANTES protein was determined by immunocytochemistry in cytocentrifuge preparations of BAL cells. The percentage of RANTES immunopositive BAL cells was obtained by counting a minimum of 200 cells $\cdot$ slide $^{-1}$. The data shown are individual results and group medians (horizontal bars). RANTES: regulated on activation, normal T-cell expressed and secreted; BAL: bronchoalveolar lavage; FA: fibrosing alveolitis.
The contribution of the individual cell types to overall RANTES protein expression was distinct in FA and sarcoidosis (fig. 2). In sarcoidosis, alveolar macrophages, defined by morphological criteria and the expression of the CD68 surface marker, appeared to be the major source of RANTES protein: $92 \%$ of RANTES+ BAL cells were macrophages. The contribution of alveolar macrophages to overall RANTES expression in FA was substantially lower, and, interestingly, a significant proportion $(32 \%)$ of RANTES+ cells were eosinophils. The contribution of T-lymphocytes (CD2+ cells) to RANTES immunopositivity in all three groups was substantially lower (median $0.2 \%$, range $0-6 \%$ of overall expression). Representative examples of RANTES protein expression in the BAL cells are shown in figures 3 and 4 . The
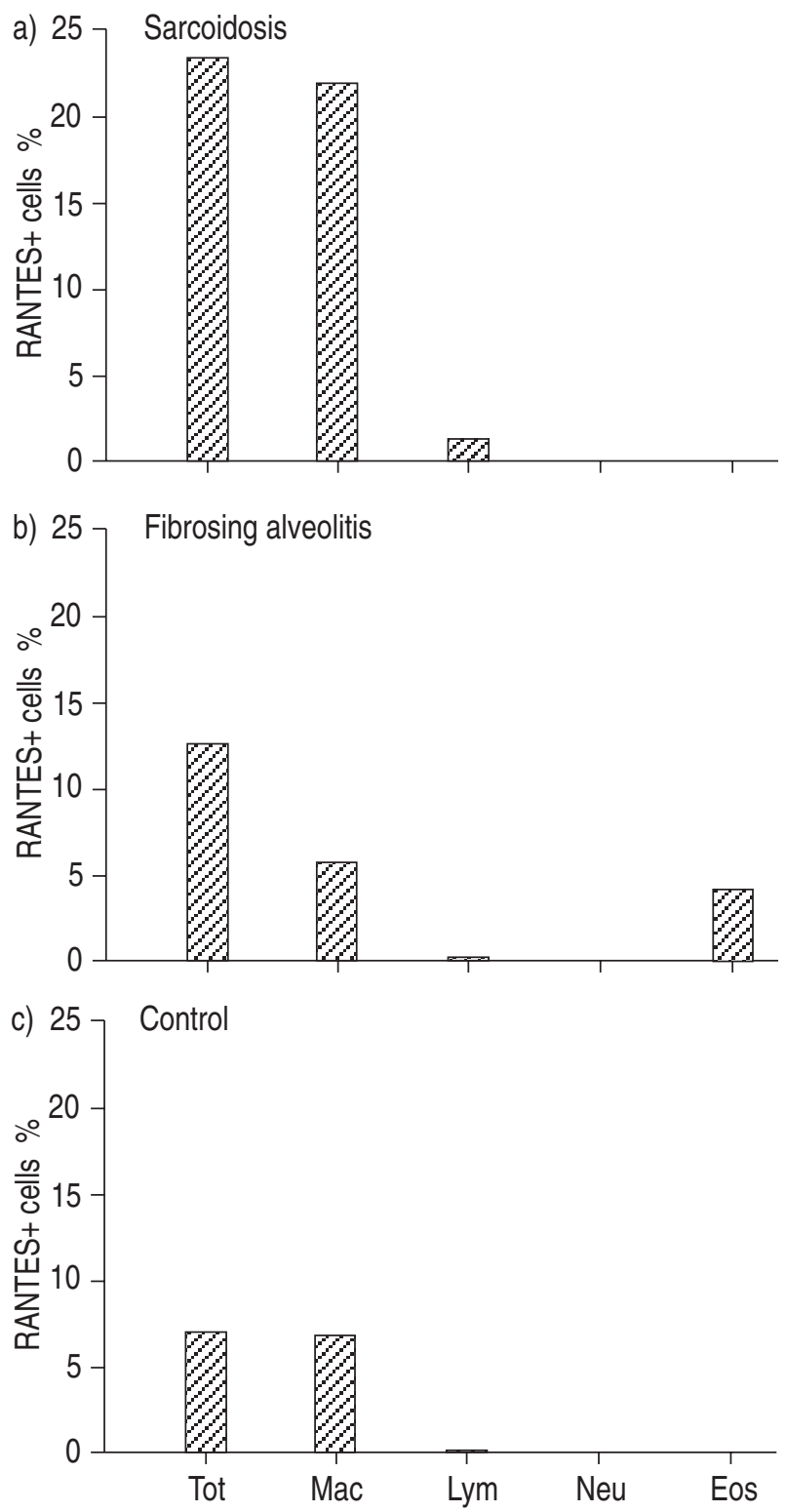

Fig. 2. - RANTES protein expression in individual cells types. The data show the contribution of individual cells types to the total percentage of RANTES positivity: a) in patients with sarcoidosis; b) in patients with fibrosing alveolitis; and c) controls. Median values are presented. Tot: total BAL cells; Mac: macrophages; Lym: lymphocytes; Neu: neutrophils; Eos: eosinophils. For further definitions see legend to figure 1 . 

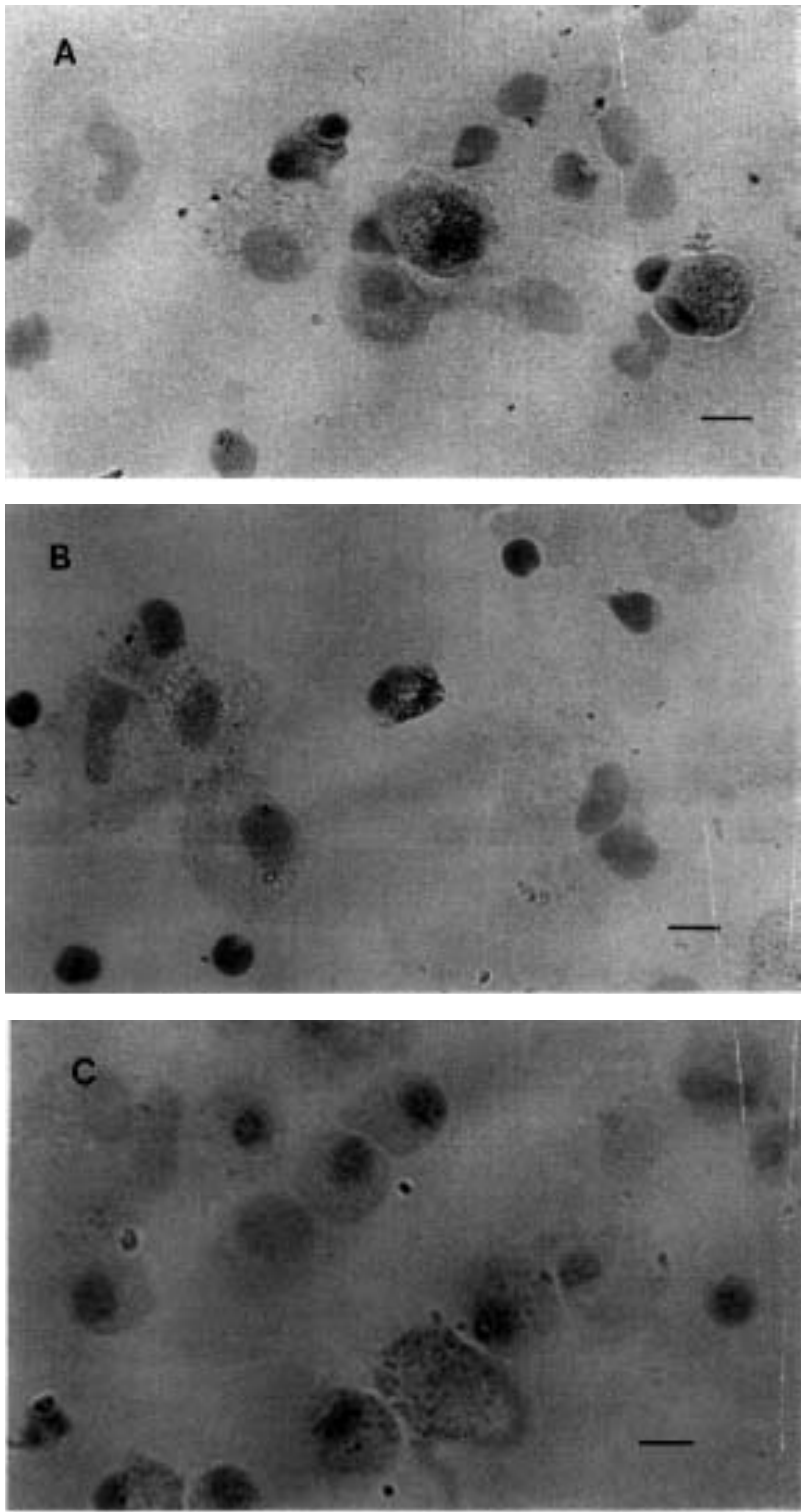

Fig. 3. - Detection of RANTES protein in BAL cells. Positively stained cells (red colour) were observed in BAL cytospin preparations incubated with anti-RANTES antibody (A and B), but not with the irrelevant control antibody (C). RANTES protein was localized to the cytoplasm of macrophages (A) and eosinophils (B). For definitions see legend to figure 1. (Avidin-biotin enzyme complex method with 3-amino-9-ethyl carbazole as substrate and counterstained with haematoxylin; internal scale bar $=10 \mu \mathrm{m}$ ).

latter figure compares the patterns of RANTES, CD68 and CD2 immunoreactivity in the lavage cells of a patient with sarcoidosis, and demonstrates that the RANTES+ cells are not lymphocytes. Further support for this comes from morphometry. The diameters of the CD68+ cells (macrophages) ranged 12.2-30.5 $\mu \mathrm{m}$ (mean $\pm \mathrm{sD}, 20.9 \pm 3.8$ $\mu \mathrm{m})$, and the majority of the RANTES+ cells were in this size range. By contrast, the range of sizes of CD2+ cells (lymphocytes) was 6.2-9.1 $\mu \mathrm{m}(7.7 \pm 0.6 \mu \mathrm{m})$, and only $8 \%$ of RANTES+ cells were of this size (data not shown).

The expression of RANTES protein was localized in the cytoplasm, rather than membrane-associated, because permeabilization of cell membranes was necessary prior to immunocytochemistry to obtain staining. The appearance of RANTES expression in eosinophils
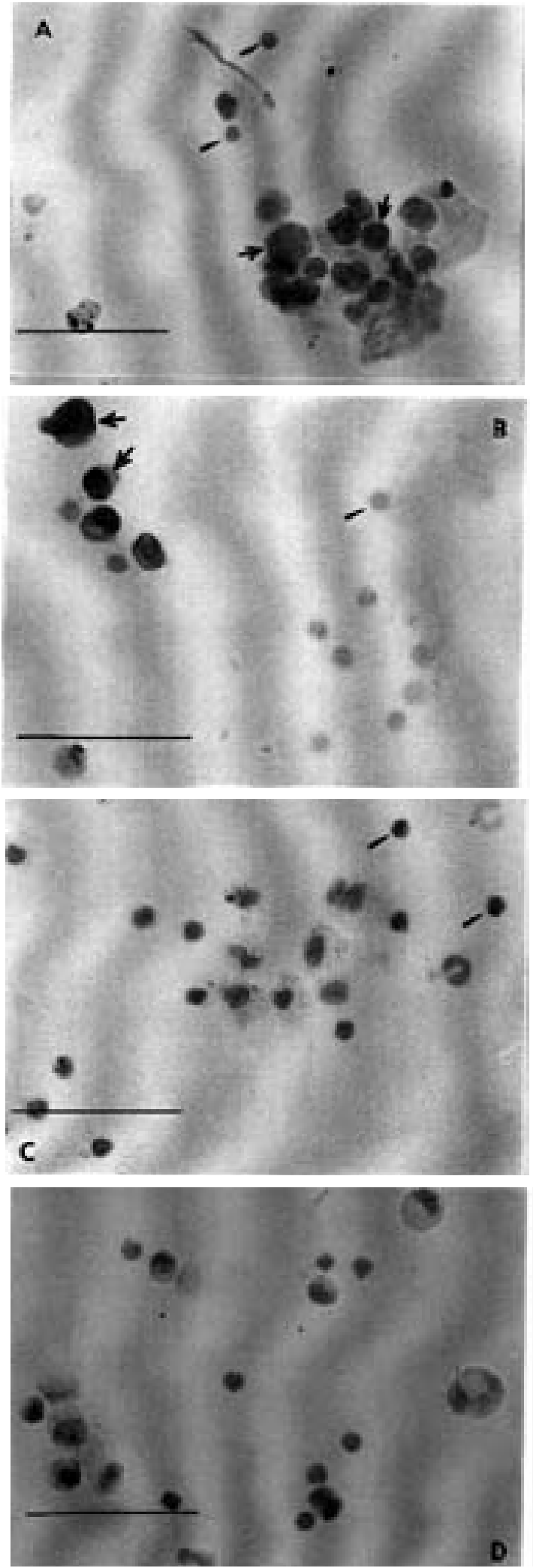

Fig. 4. - Identification of RANTES-positive cells. Cell types expressing RANTES were defined by staining patterns with anti-CD68 (macrophages) and anti-CD2 (lymphocytes). Examples of the staining patterns are shown. A) RANTES; B) CD68; C) CD2; D) negative isotype-matched control. Note that RANTES+ cells are of the morphology of CD68+ cells seen in B, and not that of the CD2+ cells, seen in C. RANTES: regulated on activation, normal T-cell expressed and secreted. (internal scale bar $=10 \mu \mathrm{m}$ ). 
was predominantly granular, suggesting that RANTES is eosinophil granule-associated.

\section{RANTES $m R N A$ expression in the lower respiratory tract cells}

RT-PCR analysis. RANTES mRNA was detected in unseparated BAL cells from all 39 patients and control subjects. Semiquantitative analysis of RANTES mRNA expression, utilizing RANTES/ $\beta$-actin ratio (ODR), showed that RANTES transcripts were upregulated in patients with sarcoidosis (fig. 5). All sarcoidosis patients had an ODR higher than 0.2 , by comparison with 11 of 20 patients with FA and 4 of 13 controls. The difference in RANTES mRNA expression between sarcoidosis patients and the control group, and also between sarcoidosis patients and the FA group was significant ( $p=0.02$ and $p=0.04$, respectively). In the FA group, more individuals expressed higher levels of RANTES mRNA than in the control group: $55 \%$ of FA subjects had ODR higher than 0.2 compared with $31 \%$ controls, but the difference between the groups was not significant.

\section{RANTES mRNA expression assessed by in situ hybridiza-} tion

To confirm that the RANTES protein, which is localized to the cytoplasm of the BAL cells, is synthesized by these cells and not taken up from the local milieu, ISH with RANTES specific oligonucleotide probes was performed on BAL cell cytospins. In lavage samples obtained from the sarcoidosis and FA patients, RANTES mRNA expression was found almost exclusively in alveolar macrophages and eosinophils (fig. 6). Lymphocytes rarely expressed RANTES mRNA: in all samples less than $5 \%$ of lymphocytes contained RANTES mRNA.

\section{RANTES expression and BAL inflammatory cells}

Lymphocytes and eosinophils in the lower respiratory tract. There was a significant difference $(\mathrm{p}=0.003)$ between the percentages of BAL lymphocytes obtained from the group of sarcoidosis patients and from the control group (median values $24 \%$, range $10-28 \%$; and $4 \%$, $2-21 \%$, respectively). The FA group and controls did not differ significantly in percentages of BAL lymphocytes (median 8\%, range $1-61 \%$; and $4 \%, 2-21 \%$, respectively). Regardless of the proportion of lymphocytes in the lavage return, the great majority (median $73 \%$, range $62-89 \%$ ) of BAL lymphocytes in all patients and control subjects expressed CD45RO. The percentage of BAL cells which were CD45RO+ (differential CD45RO+ lymphocyte count) ranged $0.6-54 \%$, and total CD45RO+ lymphocyte numbers ranged $0.1 \times 10^{6}$ to $27.2 \times 10^{6}$. By contrast, CD45RA immunostaining revealed that only a minor proportion of BAL lymphocytes (median $6 \%$, range 0-15\%) expressed CD45RA, consistent with the reciprocal expression of CD45RO and CD45RA on T-lymphocytes.

BAL eosinophil numbers were higher in FA (median $7 \%$, range $1.8-15.5 \%$ ) than in the other two groups (controls $0.5(0-5.0) \%$ and sarcoidosis $0.7(0-3.3) \%, \mathrm{p}=0.001)$.

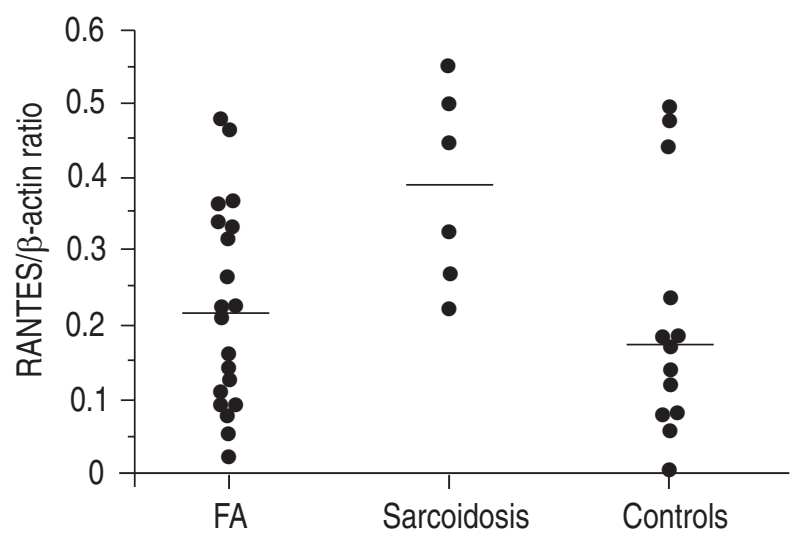

Fig. 5. - RANTES mRNA expression in unseparated lower respiratory tract cells of patients with fibrosing alveolitis $(n=20)$, sarcoidosis $(n=6)$, and controls $(n=13)$. The RANTES/ $\beta$-actin optical density ratios (ODRs), obtained by normalizing optical density (OD) measurements of RANTES amplification products to the OD values of corresponding $\beta$-actin PCR products are shown. Data are presented as individual results and group medians (horizontal bars). mRNA: messenger ribonucleic acid. For further definitions see legend to figure 1 . Difference between sarcoidosis and controls, $\mathrm{p}=0.02$; difference between sarcoidosis and FA, $\mathrm{p}=0.04$.
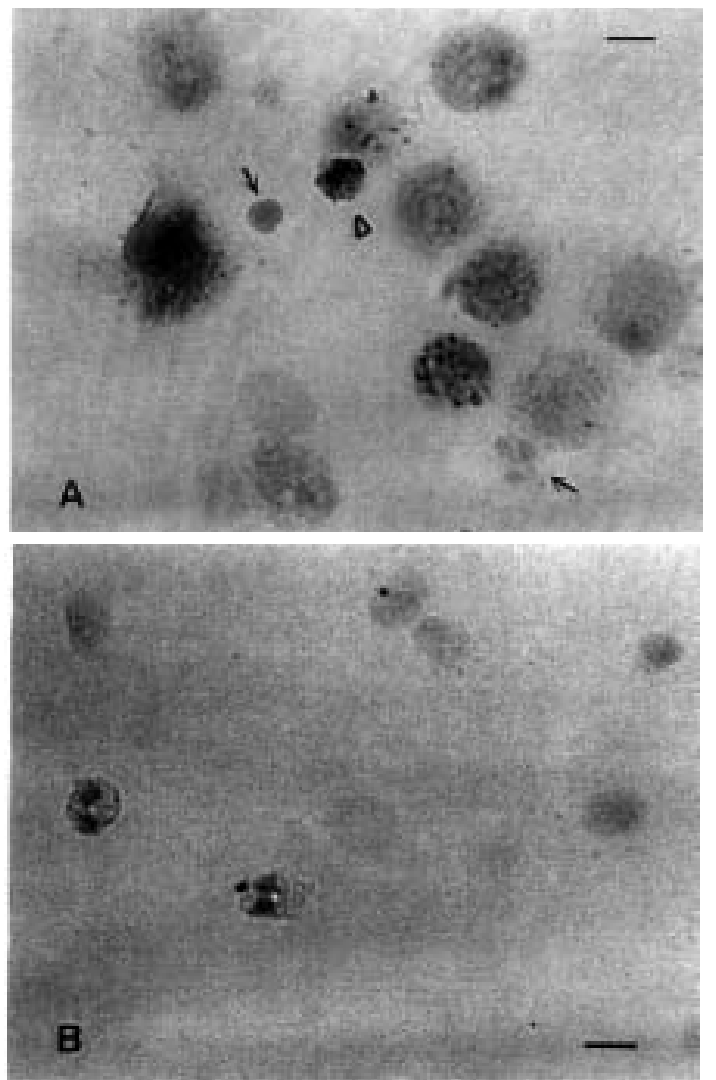

Fig. 6. - Detection of RANTES messenger ribonucleic acid (mRNA) in BAL macrophages and eosinophils by in situ hybridization. After hybridization of the digoxigenin-labelled oligonucleotide probe, positive signals were obtained in the cytoplasm of alveolar macrophages and the eosinophil (A, triangle). Note the absence of mRNA in the lymphocyte and the neutrophil (arrows). No signal was detected in the negative control (B), which was pretreated with ribonuclease (RNAse) to confirm the specificity of the probe for ribonucleic acid (RNA) and to exclude nonspecific reactions during the detection. For definitions see legend to figure 1. (Digoxigenin labelled hybridized probes, with anti-digoxigenin, nitroblue tetrazolium (NBT)/5-bromo4-chloro-3-indolyl phosphate (BCIP) substrate and nuclear red counterstaining; internal scale bar $=10 \mu \mathrm{m})$. 
Relationship between RANTES expression and the number of lymphocytes and eosinophils lavaged from the lower respiratory tract

Because RANTES is a lymphocyte and eosinophil attractant, the possible relationship between RANTES expression and the number of its potential target cells lavaged from the LRT was investigated.

There was a clear association between the number of BAL lymphocytes and RANTES mRNA and protein expression (fig. 7). In subjects with an increased number of lymphocytes in BAL (>13\%), RANTES mRNA and protein expression was significantly higher than in the subjects with normal BAL lymphocyte numbers $(<13 \%)(\mathrm{p}=0.02$ (mRNA); and $\mathrm{p}=0.04$ (protein)).

This was further explored by correlating RANTES/ $\beta$ actin ratio (ODR) with the percentage and total number of lymphocytes, macrophages, neutrophils and eosinophils obtained by BAL. Of these variables, BAL differential lymphocyte count showed a positive correlation
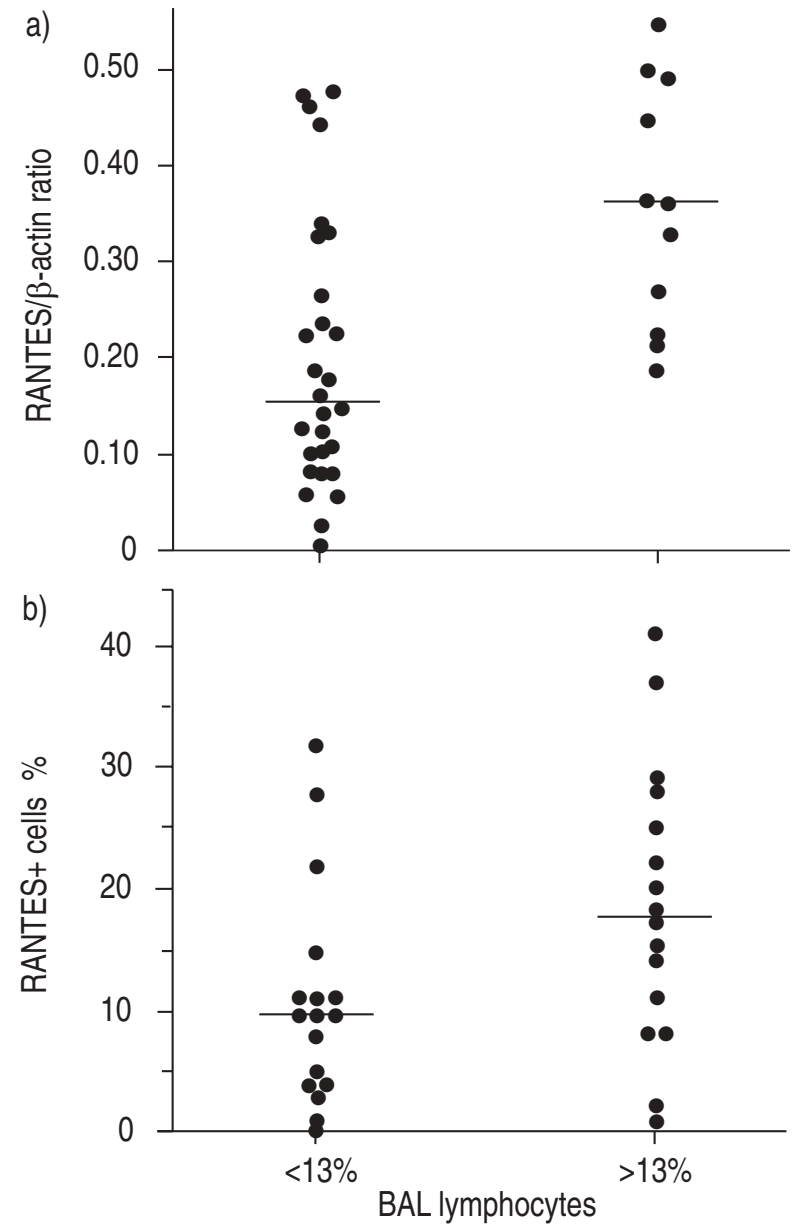

Fig. 7. - A comparison of RANTES expression in subjects with normal and abnormal BAL lymphocyte differential counts. The subjects were separated into two groups on the basis of differential lymphocyte count $(>13 \%$ or $<13 \%)$ and related to RANTES mRNA and protein expression. Data are presented as individual results and group medians (horizontal bars). a) RANTES mRNA expression (ODR), $\mathrm{p}=0.02$ (BAL lymphocytes $<13 \% \quad(\mathrm{n}=28)$, BAL lymphocytes $>13 \%$ $(\mathrm{n}=11))$. b) RANTES protein expression (\% of RANTES+ BAL cells), $\mathrm{p}=0.04$ (BAL lymphocytes $<13 \%(\mathrm{n}=17)$, BAL lymphocytes $>13 \%$ $(\mathrm{n}=16))$. ODR: optical density ratio; mRNA: messenger ribonucleic acid. For further definitions see legend to figure 1 . $\left(r_{s}=0.63 ; p=0.0001\right)$ with the ODR (fig. 8a). The same relationship was observed when comparisons were made between ODR and total BAL cell numbers. The numbers of lavaged eosinophils and neutrophils did not correlate with RANTES mRNA expression ( $\mathrm{p}>0.1)$.

Importantly, RANTES mRNA expression was low (median 0.083, range 0.006-0.179) in the control subjects
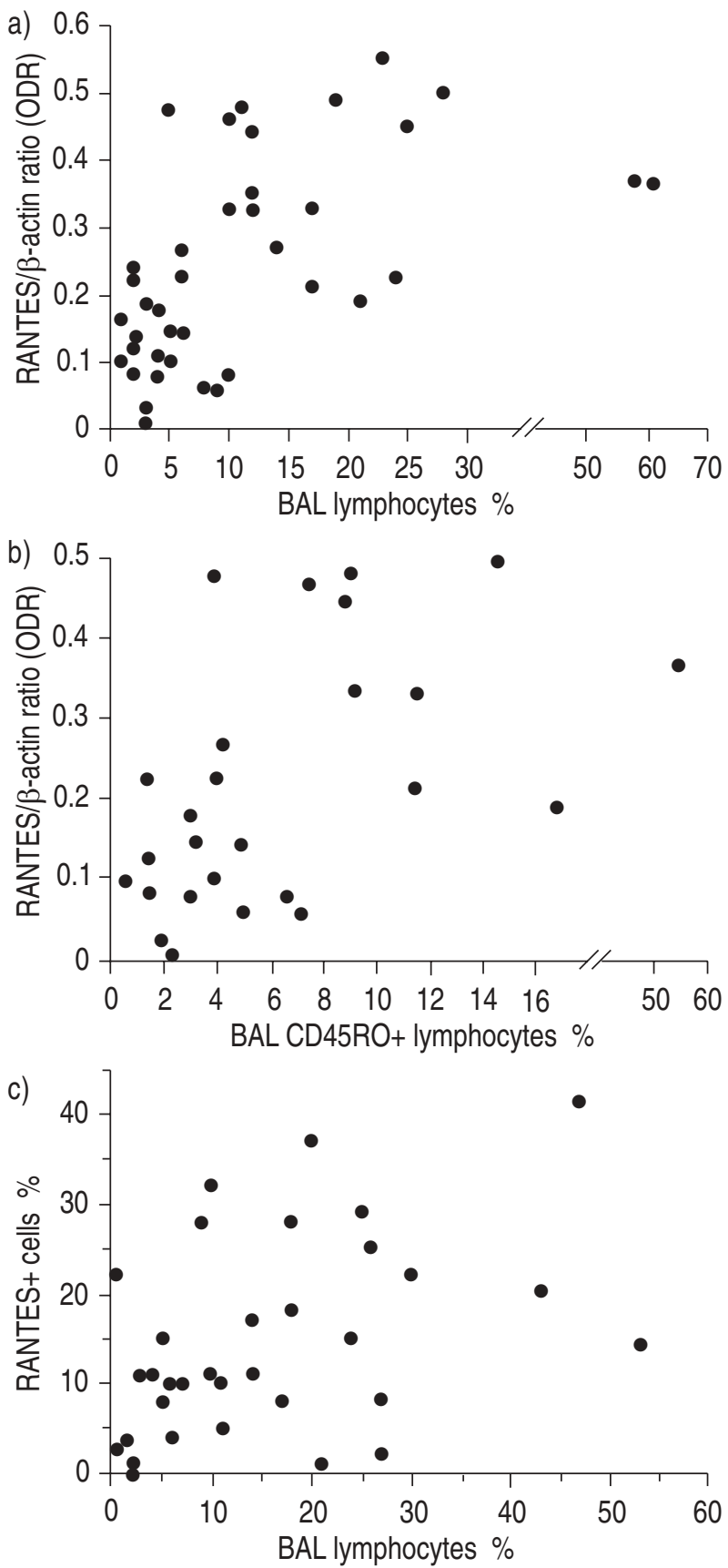

Fig. 8. - Correlation between RANTES expression and BAL lymphocyte counts. The correlations were calculated using 39 subjects for association between mRNA and lymphocyte counts, 26 subjects for association between mRNA and CD45RO+ lymphocyte counts, and 33 subjects for correlation of the RANTES protein and lymphocyte counts. Individuals with and without disease were included. a) ODR versus BAL differential lymphocyte count (Spearman's rank correlation coefficient $\left.\left(\mathrm{r}_{\mathrm{s}}\right)=0.63 ; \mathrm{p}=0.0001\right)$; b) ODR versus BAL differential CD45RO+ lymphocyte count $\left(\mathrm{r}_{\mathrm{s}}=0.57 ; \mathrm{p}=0.001\right)$; $)$ protein expression versus BAL differential lymphocyte count $\left(\mathrm{r}_{\mathrm{s}}=0.39 ; \mathrm{p}=0.03\right)$. For definitions see legend to figures 1 and 7. 
with $<4 \%$ lymphocytes in lavage, by comparison with $0.316(0.059-0.492)$ when lymphocyte percentages were $>5 \%$, providing further support for the concept of RANTES regulation of T-cell traffic in the normal state.

RANTES is a T-lymphocyte attractant with preference for CD45RO+ "memory" T-cells. Therefore, in a subgroup of the patients and controls, the association between RANTES mRNA expression and BAL lymphocyte subsets was analysed. There was significant correlation $\left(\mathrm{r}_{\mathrm{s}}=0.57 ; \mathrm{p}=0.001\right)$ between ODR and BAL $\mathrm{CD} 45 \mathrm{RO}+$ differential (fig. 8b) and total lymphocyte counts.

The relationship between RANTES protein expression and numbers of BAL inflammatory cells was also investigated. The percentage of RANTES positive BAL total cells showed a weak positive correlation $\left(r_{s}=0.39\right.$; $\mathrm{p}=0.03$ ), with the number of BAL lymphocytes (fig. $8 \mathrm{c}$ ), but did not correlate with neutrophils and eosinophils.

\section{Discussion}

This study has demonstrated that RANTES is expressed at the protein and mRNA level in the lower respiratory tracts of patients with interstitial lung diseases, that it is associated with the accumulation of "memory" Tcells within the lung and, importantly, that the major sites of RANTES synthesis are macrophages alone in sarcoidosis, but macrophages and eosinophils in fibrosing alveolitis. Although the data are obtained from cells recovered by BAL alone, the study suggests that RANTES is a likely potent recruiter of T-lymphocytes to the lower respiratory tract in vivo, and thus contributes to the progression of the inflammatory infiltrate in interstitial lung disease.

The investigations of RANTES mRNA and protein expression in LRT cells obtained by BAL revealed that RANTES expression was upregulated by comparison with controls in sarcoidosis, a disease characterized by a lymphocytic alveolitis, but not in FA. However, the relationship of RANTES with lymphocyte accumulation independent of disease is shown by the significant increase of RANTES expression in individuals whose lymphocyte numbers exceeded the normal range.

The semiquantification of mRNA is subject to some variability, and we have attempted to ensure the reproducibility and specificity by careful definition of experimental conditions. In this regard, a series of preliminary experiments were performed to define the optimum conditions for each primer pair, and to confirm the reproducibility of the product amount by replicate experiments on the same day and repeat amplification of the same cDNA on separate days. Furthermore, by normalization of RANTES expression data to the transcription products of the constitutively expressed $\beta$-actin gene the possibility of overinterpreting the RT-PCR results was avoided. The use of this variant of RT-PCR was substantiated by other studies [7, 21], which showed significant differences between expression of the chemokine IL-8 in cells from normal and fibrotic lung using this normalization method.

\section{RANTES in the bronchoalveolar compartment}

Knowledge of RANTES expression in vivo in human inflammatory lung disease has so far been limited to asthma and, apart from the study of WANG et al. [22], which described the presence of RANTES protein in bronchial epithelial cells, there have been no reports on RANTES expression in the bronchoalveolar compartment. In the present study, RANTES mRNA and protein were localized intracellularly mainly in alveolar macrophages, identified by morphology and CD68 positivity, and eosinophils. The predominant RANTES+ cell was the macrophage in sarcoidosis, whilst in FA there was a high number of eosinophils, which expressed RANTES mRNA and protein. These findings extend current knowledge concerning the spectrum of cells which express RANTES in the human lung, especially in the context of interstitial lung disease. Moreover, the demonstration that different cell types express RANTES in FA and in sarcoidosis supports the presumption of YING et al. [23], that cell types expressing RANTES may differ in various types of inflammation.

Human alveolar macrophages have been shown in vivo to express other chemokines, e.g. MIP-1a [8]; however, there have been no reports about their in vivo expression of RANTES. Our finding of intracellular expression of RANTES in BAL macrophages can be linked to the recent findings that these cells may release RANTES when cultivated in vitro [24, 25].

RANTES mRNA and protein have previously been detected in eosinophils isolated from peripheral blood of normal and atopic donors [26, 27], but this was not explored in lung eosinophils. In support of our findings in lung cells, LiM et al. [26] have shown that immunoreactive RANTES was localized intracellularly in eosinophils, in a granule-associated staining pattern. Moreover, we detected RANTES mRNA in eosinophils by ISH, consistent with the concept that BAL eosinophils can synthesize and store RANTES protein. Although the significance of this phenomenon is not clear, it may reflect the capability of eosinophils to play a central role in the evolution of the inflammatory component of FA, consistent with our previous studies, which showed that eosinophils are recruited to the lung at an early stage of disease defined by CT extent [28].

\section{The relationship of RANTES expression and interstitial lung disease}

The present studies of RANTES protein and mRNA expression in unseparated BAL cell pellets using RT-PCR methodology showed a correlation between RANTES mRNA expression and the number of BAL lymphocytes. This observation is in keeping with the known in vitro lymphoattractant properties of RANTES [11, 12], and is, indeed, in agreement with our initial hypothesis that RANTES attracts lymphocytes to the LRT in vivo.

The increase of RANTES expression in alveolar macrophages of patients with sarcoidosis may be relevant to the development of the T-lymphocytic alveolitis in this disease. This hypothesis is supported by reports of the localization of RANTES mRNA to macrophages within the granulomas related to sarcoidosis or tuberculosis [25]. In this context, it is interesting that Mycobacterium tuberculosis, which is considered to be a possible antigenic trigger in sarcoidosis, can induce RANTES production [29]. By contrast with sarcoidosis, patients with FA did not show a significant difference from controls 
with regard to the total number of cells expressing RANTES protein or the levels of RANTES mRNA in BAL cell pellets. However, in FA the T-cell accumulation is predominantly interstitial and fewer T-cells are present on the epithelial surface. Where they were present in high numbers, it was found that RANTES was upregulated, but this was not a disease-dependent observation, being found in control samples also. A second explanation for the difference between FA and sarcoidosis may be that sarcoidosis is the product of a type 1 T-helper (Th1) T-cell response, whereas FA involves a predominantly type 2 T-helper (Th2) type of immune response. The Th1 cytokine, interferon- $\gamma$ (IFN $\gamma$ ), which is produced by lavage [30] and interstitial [31] cells in sarcoidosis, upregulates RANTES, while the Th2 cell product, interleukin-4 (IL-4), inhibits RANTES [32]. We [33] and others [31] have previously shown that the cytokine expression in FA is predominantly of Th2 pattern.

Nonetheless, our data suggest that RANTES has a role in T-cell accumulation in both the diseases studied, and is upregulated most in sarcoidosis, which is a classical T-cell mediated disorder. The fact that RANTES was found to be expressed in the normal lung may implicate this cytokine in the recruitment of lymphocytes to the lung as a part of normal host defence (immune surveillance).

In conclusion, we have demonstrated that the chemokine RANTES is implicated in the attraction of Tlymphocytes to the lower respiratory tract, and that the upregulation of RANTES messenger ribonucleic acid and protein expression is relevant to the development of the lymphocytic alveolitis in sarcoidosis, and possibly to further propagation of the inflammatory infiltrate in fibrosing alveolitis. Further studies of the contribution of RANTES to the pathogenesis of interstitial lung disease should involve determination of functional activity of RANTES protein in bronchoalveolar lavage fluid, and should address the question of differential expression of RANTES at different stages of disease.

Acknowledgements: The authors thank P. Balharova for technical assistance and V. Lichnovsky for help with photomicrography.

\section{References}

1. du Bois RM, Holroyd KJ, Saltini C, Crystal RG. Granulomatous processes. In: Crystal RG, West JB, eds. The Lung: Scientific Foundations. New York, Raven Press, 1991, 1925-1938.

2. Hol BEA, Hintzen RQ, Van Lier RAW, Alberts C, Out TA, Jansen HM. Soluble and cellular markers of T-cell activation in patients with pulmonary sarcoidosis. Am Rev Respir Dis 1993; 148: 643-649.

3. Allen JN, Davis WB. Eosinophilic lung diseases. Am J Respir Crit Care Med 1994; 150: 1423-1438.

4. du Bois RM. Fibrosing alveolitis. Апnи Rev Med 1993; 44: 441-450.

5. Wells AU, Lorimer S, Majumdar S, et al. Fibrosing alveolitis in systemic sclerosis: increase in memory Tcells in lung interstitium. Eur Respir J 1995; 8: 266-271.

6. Schall TJ, Bacon KB. Chemokines, leukocyte trafficking, and inflammation. Curr Opinion Immunol 1994; 6: $865-873$.
7. Southcott AM, Jones $\mathrm{KP}$, Li D, et al. Interleukin-8 expression: differences between lone fibrosing alveolitis and fibrosing alveolitis in systemic sclerosis. Am J Respir Crit Care Med 1995; 151: 1604-1612.

8. Standiford TJ, Rolfe MW, Kunkel SL, et al. Macrophage inflammatory protein-1a expression in interstitial lung disease. J Immunol 1993; 151: 2852-2863.

9. Car BD, Meloni F, Luisetti M, Semenzato G, GialdroniGrassi G, Walz A. Elevated IL-8 and MCP-1 in the bronchoalveolar lavage fluid of patients with idiopathic pulmonary fibrosis and pulmonary sarcoidosis. Am J Respir Crit Care Med 1994; 149: 655-659.

10. Schall TJ, Jongstra J, Dyer BJ, et al. A human T-cell specific molecule is a member of a new gene family. $J$ Immunol 1988; 141: 1018-1025.

11. Schall TJ, Bacon K, Toy KJ, Goeddel DV. Selective attraction of monocytes and T-lymphocytes of the memory phenotype by cytokine RANTES. Nature 1990; 347: 669-671.

12. Taub DD, Conlon K, Lloyd AR, Oppenheim JJ, Kelvin DJ. Preferential migration of activated CD4+ and CD8+ T-cells in response to MIP-1a and MIP-1b. Science 1993; 260: 355-358.

13. Alam R, Stafford S, Forsythe P, et al. RANTES is a chemotactic and activating factor for human eosinophils. J Immunol 1993; 150: 3442-3448.

14. Cordell JL, Falini B, Erber WN, et al. Immunoenzymatic labelling of monoclonal antibodies using immune complexes of alkaline phosphatase and monoclonal antialkaline phosphatase (APAAP complexes). J Histochem Cytochem 1984; 32: 219-229.

15. Chomczynski P, Sacchi N. Single-step method of RNA isolation by acid guanidium thiocyanate-phenol-chloroform extraction. Anal Biochem 1987; 162: 156-159.

16. Krug MS, Berger SL. First-strand cDNA synthesis primed with oligo(dT). Methods Enzymol 1987; 152: 316-325.

17. Corrochano LM. A test of human cDNA synthesis by the polymerase chain reaction. Genet Anal Tech Appl 1991; 8: 134-135.

18. Ponte P, Ng SY, Engel J, Gunning P, Kedes L. Evolutionary conservation in the untranslated regions of actin mRNAs: DNA sequence of a human beta-actin cDNA. Nucleic Acids Res 1984; 12: 1687-1696.

19. Trapnell BC. Quantitative evaluation of gene expression in freshly isolated human respiratory epithelial cells. Am J Physiol 1993; 264 (Lung Cell Mol Physiol 8): L199-L212.

20. Heeger P, Wolf G, Meyers C, et al. Isolation and characterization of cDNA from renal tubular epithelium encoding murine RANTES. Kidney Int 1992; 41: 220-225.

21. Carré PC, Mortenson RL, King TE, Noble PW, Sable CL, Riches DWH. Increased expression of the interleukin- 8 gene by alveolar macrophages in idiopathic pulmonary fibrosis: a potential mechanism for the recruitment and activation of neutrophils in lung fibrosis. J Clin Invest 1991; 88: 1802-1810.

22. Wang JH, Devalia JL, Xia CL, Sapsford RJ, Davies RJ. Expression of RANTES by human bronchial epithelial cells in vitro and in vivo and the effect of corticosteroids. Am J Respir Cell Mol Biol 1996; 14: 27-35.

23. Ying S, Taborda-Barata L, Meng Q, Humbert M, Kay $\mathrm{AB}$. The kinetics of allergen-induced transcription of messenger RNA for monocyte chemotactic protein-3 and RANTES in the skin of human atopic subjects: relationship to eosinophil, T-cell, and macrophage recruitment. J Exp Med 1995; 181: 2153-2159.

24. Folkard SG, Westwick J, Millar AB. Production of IL8 and RANTES from macrophages and monocytes of 
moderate asthmatic subjects. Abstracts of the IIIrd Conference on Chemokines, Bath, England, 28-30 June 1995; p.10.

25. Devergne O, Marfaing-Koka A, Schall TJ, et al. Production of the RANTES chemokine in delayed-type hypersensitivity reactions: involvement of macrophages and endothelial cells. $J$ Exp Med 1994; 179: 1689-1694.

26. Lim KG, Wan HC, Bozza PT, et al. Human eosinophils elaborate the lymphocyte chemoattractants IL-16 (lymphocyte chemoattractant factor) and RANTES. $J$ Immunol 1996; 156: 2566-2570.

27. Ying S, Meng Q, Taborda-Barata L, Corrigan JC, et al. Human eosinophils express messenger RNA encoding RANTES and store and release biologically active RANTES protein. Eur J Immunol 1996; 26: 70-76.

28. Wells AU, Hansell DM, Cailes J, et al. Bronchoalveolar lavage (BAL) cellularity in fibrosing alveolitis: a comparison between lone cryptogenic fibrosing alveolitis (CFA) and the fibrosing alveolitis of systemic sclerosis (FASSc). (Abstract). Eur Respir J 1995; 8: 453s

29. Kwon OJ, Kim HJ, Au BT, et al. RANTES and IL-8 were expressed in airway epithelial cells in response to tubercle bacilli stimulation. Am J Respir Crit Care Med 1996; 153: A130.

30. Robinson BWS, McLemore TL, Crystal RG. Gammainterferon is spontaneously released by alveolar macrophages and lung T-lymphocytes in patients with pulmonary sarcoidosis. J Clin Invest 1985; 75: 1488-1495.

31. Wallace WAH, Ramage EA, Lamb D, Howie SEM. A type-2 (Th2-like) pattern of immune response predominates in the pulmonary interstitium of patients with cryptogenic fibrosing alveolitis (CFA). Clin Exp Immunol 1995; 101: 436-441.

32. Marfaing-Koka A, Devergne O, Gorgone G, et al. Regulation of the production of the RANTES chemokine by endothelial cells: synergistic induction by IFN- $\gamma$ plus TNF- $\alpha$ and inhibition by IL-4 and IL-13. J Immunol 1995; 154: 1870-1878.

33. Hamid Q, Majumdar S, Sheppard M, et al. Expression of IL4, IL-5, IFN- $\gamma$, and IL-2 mRNA in fibrosing alveolitis associated with systemic sclerosis. Am Rev Respir Dis 1993; 147: A479. 